\title{
Monitoring strategies for patients treated with the new oral anticoagulants and the need for laboratory evaluation of hemostasis
}

\author{
Andrea Fontanella, Pierpaolo Di Micco \\ Department of Medicine, Buon Consiglio Fatebenefratelli Hospital, Naples, Italy
}

\begin{abstract}
New oral anticoagulants that directly inhibit Factor IIa (dabigatran) or Factor Xa (rivaroxaban, apixaban) are currently available for prevention of venous thromboembolism (VTE) after orthopedic surgery, treatment of acute VTE, and prevention of arterial thromboembolism in non-valvular atrial fibrillation. These agents offer advantages over vitamin $\mathrm{K}$ antagonists including rapid onset, shorter half-lives, fewer drug interactions, and the lack of a need for routine monitoring. The fact that monitoring is not required should not, however, lead to lack of surveillance or a fire and forget medicine approach because there are several medical conditions that require careful clinical surveillance and, sometimes, laboratory monitoring. The main situations that require close monitoring are major bleeding, assessment of compliance (in particular during comorbidities other than vascular disease, e.g. dementia), overdose, sudden or progressive renal dysfunction, extreme body weight, concomitant use of other drugs that may induce impairment of new oral anticoagulants, need for urgent surgery.
\end{abstract}

\section{Introduction}

\section{Goals of antithrombotic treatment with new oral anticoagulants and historical background}

The best known and most commonly used anticoagulant drugs include the heparins (unfractionated heparin or low molecular weight heparins or fondaparinux) and vitamin $\mathrm{K}$ antagonists (VKAs). Al-

Correspondence: Pierpaolo Di Micco, Department of Medicine, Buon Consiglio Fatebenefratelli Hospital, via Manzoni 220, 801123 Naples, Italy.

Tel.: +39.339.8078.146 - Fax: +39.081.5981.385

E-mail: pdimicco@libero.it

Key words: new oral anticoagulant, hemostasis, laboratory, monitoring.

Contributions: the authors contributed equally.

Conflict of interests: the authors declare no potential conflict of interests.

Received for publication: 27 April 2013.

Revision received: 5 July 2013.

Accepted for publication: 24 June 2013.

This work is licensed under a Creative Commons Attribution NonCommercial 3.0 License (CC BY-NC 3.0).

CCopyright A. Fontanella and P. Di Micco, 2013

Licensee PAGEPress, Italy

Italian Journal of Medicine 2013; 7(s8):59-64

doi:10.4081/itjm.2013.s8.59 though the mechanisms of these drugs vary greatly, they do share one common characteristic: they act all as indirect drugs. Vitamin $\mathrm{K}$ antagonists act by reducing the synthesis of clotting factors so reducing the power of clotting ability. ${ }^{1}$ Heparins act through their link with antithrombin so inducing an increased and fast inhibition of activated clotting factors. ${ }^{2}$ The efficacy of these drugs has been shown in various reports over recent years, and also through clinical trials, evidence-based medicine and international guidelines. ${ }^{3}$

Recently, new families of anticoagulant drugs have been developed with different pharmacodynamic and pharmacokinetic profiles. Among these we can distinguish drugs that directly target against Factor Xa or IIa. Of course, direct action toward activated clotting factors are really promising. From this point of view, absorption, metabolism, elimination and interactions with food or other drugs are also different for direct Xa inhibitors and IIa inhibitors if compared with vitamin K antagonists and/or heparins (Table 1). ${ }^{4}$ These pharmacological characteristics of new anticoagulants facilitate the daily clinical administration of food or other drugs in the presence of comorbidities, even if clinical experience regarding food interactions is currently limited.

The pharmacological action of classic anticoagulants (i.e. vitamin $\mathrm{K}$ antagonists and heparins) is monitored through international normalized ratio (INR) values for vitamin $\mathrm{K}$ antagonists by aPTT ratio for unfractionated heparins and by levels of inactivated $\mathrm{Xa}$ factor for low molecular weight heparins/fondaparinux, while such monitoring is usually not required for new oral anticoagulants, such as direct $\mathrm{Xa}$ in- 
hibitors (i.e. rivaroxaban, apixaban, edoxaban and others) or IIa inhibitors (i.e. dabigatran) based also on a relatively short half-life.

However, not monitoring should mean do not monitor prothrombin time (PT)-INR or aPTT, but it does not mean do not oversee the situation or fire and forget medicine! This concept should always be kept in mind in new approaches to anticoagulant treatments to ensure a correct strategy is adopted concerning thrombotic disease, patients and drugs. Moreover, in this field, several trials study the safety and efficacy of treatment with new oral anticoagulants for prevention of cardioembolic stroke in patients with atrial fibrillation or for prevention and treatment of venous thromboembolism. However, the anticoagulant activity of new oral anticoagulants can be monitored in rare cases by several laboratory tests, even if these have not been standardized or are not routinely available.

\section{Clinical conditions with indication to monitor new oral anticoagulants}

Prescription of this new class of drugs requires vigilance, also because this is a fragile patient population and new oral anticoagulants (NOACs) are drugs with potentially severe complications. Patients should return on a regular basis for ongoing review of their treatment, preferably every three months. This may be carried out by general practitioners with experience in this field and/or by appropriate secondary care physicians.

Renal function, a predictor of bleeding with anticoagulant drugs in general, should be assessed prior to initiation of therapy with novel oral anticoagulants and monitored during treatment, much more frequently than in patients with chronic renal dysfunction. Monitoring is particularly important for dabigatran, which is excreted by the kidneys. ${ }^{5}$

In accordance with recent EHRA2013 guidelines, ${ }^{6}$ monitoring should be performed according to this scheme:

- Yearly: hemoglobin, renal and liver function

-6-monthly: renal function if creatinine clearance (preferably measured by the Cockroft method) $30-60 \mathrm{~mL} / \mathrm{min}$, or if on dabigatran and $>75$ years or fragile

-3-monthly: if creatinine clearance $15-30 \mathrm{~mL} / \mathrm{min}$ - on indication: if intercurring condition that may impact renal or hepatic function.

Because their direct antithrombotic activity is due to their pharmacodynamic profile, routine monitoring of new oral anticoagulants is not indicated either for IIa inhibitor or for Xa inhibitors. Moreover, the rate of complications in reported studies was very low for all drugs so routine monitoring of new oral anticoagulants is not indicated from a clinical point of view.

Therefore, clinical conditions in which new oral anticoagulants are monitored are similar to those clin- ical conditions that also indicate monitoring of the classic anticoagulants (i.e. anti-vitamin $\mathrm{K}$ drugs and heparins), such as when a severe life-threatening complication is present or possible. Clinical conditions in which monitoring of new oral anticoagulants is suggested may be summarized as follows: major bleeding (in particular, sudden bleeding or major bleeding with recent intake of new oral anticoagulants), thrombotic events, assessment of compliance (i.e. in particular, during comorbidities other than vascular disease, e.g. dementia), overdose, sudden or progressive renal dysfunction, extreme body weight, concomitant use of other drugs that may induce impairment of new oral anticoagulants, need for urgent surgery.

So, principally, monitoring of new oral anticoagulants is a clinical monitoring, with particular attention to the conditions listed above.

However, specific monitoring of anticoagulant activity of new oral anticoagulants may be required for patients with sudden kidney failure or with progressive renal dysfunction with creatinine clearance less than $20 \mathrm{~mL}$, particularly in conjunction with a surgical approach.

Major bleeding is always a life-threatening disease or complication, and monitoring the anticoagulant activity of any anticoagulant drug may help clinical management, even though a specific antidote is not available, and often it may not be required to restore blood flow or to escape further bleeding complications.

Emergency situations (e.g. hemorrhage, overdose, urgent surgery/invasive procedure) necessitate rapid assessment of coagulation status. This clinical approach may be helpful both for sudden bleeding and for recent drug intake or over dosage, or for patients at risk of bleeding who are non-compliant. On the other hand, patients with extreme body weight and patients who are taking several drugs may have a different distribution of new oral anticoagulants, in particular Xa inhibitors, respectively for different metabolic response and pharmacokinetic and/or pharmacodynamic interactions (Tables 1 and 2). ${ }^{4,8}$ Modest changes in the use of IIa inhibitors are needed for extreme body weight; however, caution is needed in cases of low body mass index, especially if associated to renal impairment.

However, conventional coagulation assays have limitations when used to measure novel oral anticoagulant effect. There is, in fact, no precise relationship between the routine coagulation tests available and the anticoagulant activity (Table 3 ). ${ }^{9}$

\section{Pharmacological basis of reduced monitoring of new anticoagulants}

\section{Xa inhibitors}

Rivaroxaban, apixaban and edoxaban are direct, reversible, competitive inhibitors of free Xa factor. Rivaroxaban has a half-life of 5-9 h, while apixaban has a 

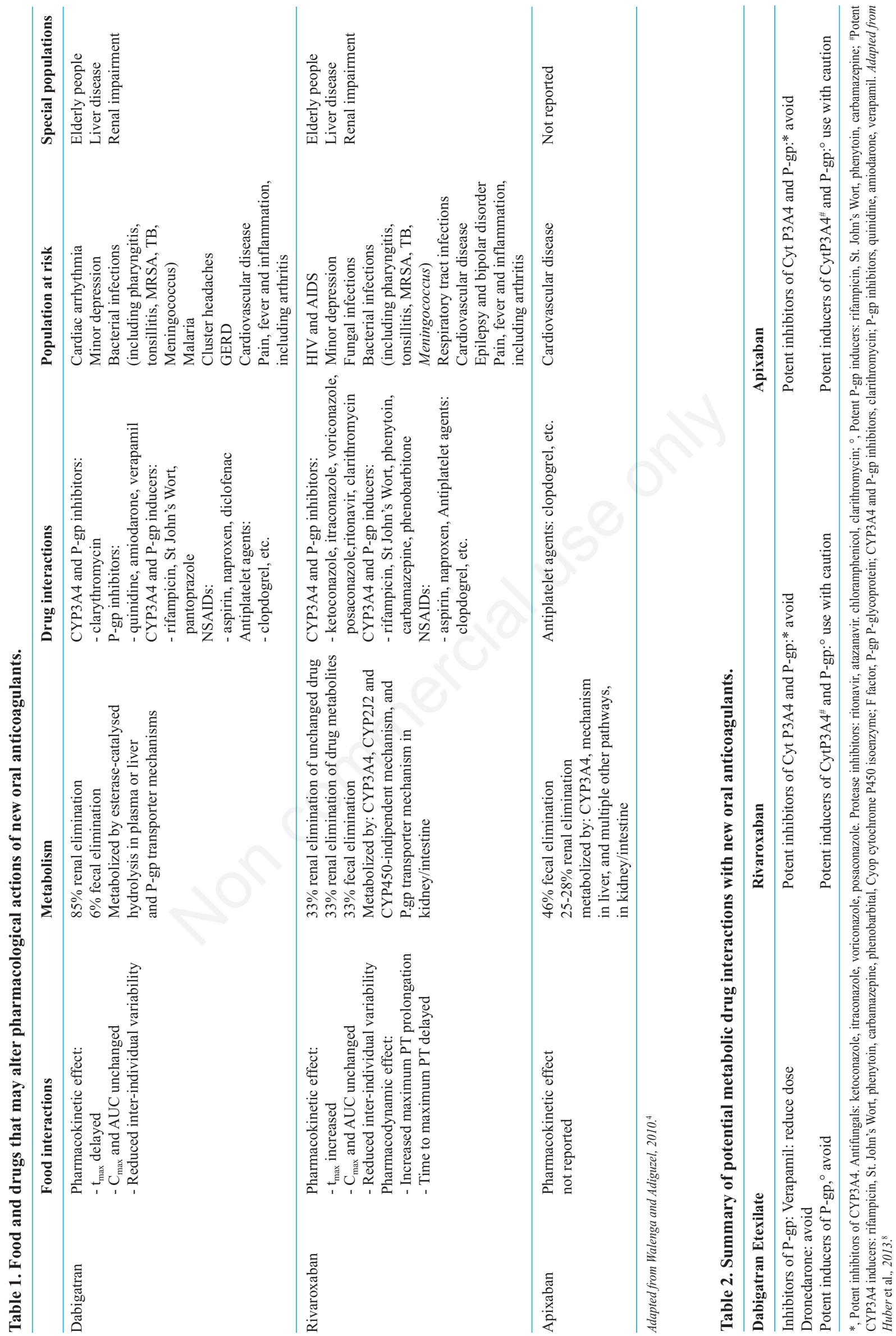

[Italian Journal of Medicine 2013; 7(s8):e8] 
Table 3. Effect of new oral anticoagulants on commonly used coagulation tests.

\begin{tabular}{|c|c|c|c|c|c|c|c|}
\hline & $\begin{array}{l}\text { Prothrombin } \\
\text { time (PT) }\end{array}$ & $\begin{array}{c}\text { Activated } \\
\text { partial } \\
\text { thromboplastin } \\
\text { time (PTT) }\end{array}$ & $\begin{array}{c}\text { Thrombin } \\
\text { clotting } \\
\text { time (CTT) }\end{array}$ & $\begin{array}{l}\text { Ecarin } \\
\text { clotting time } \\
(\text { ECT })\end{array}$ & $\begin{array}{c}\text { Hemoclot } \\
\text { assay }\end{array}$ & \multicolumn{2}{|c|}{$\begin{array}{l}\text { Anti-Factor } \\
\text { Xa activity } \\
\text { Clot based } \\
\text { chromogenic }\end{array}$} \\
\hline Dabigatran & $\begin{array}{c}\text { } \text { or no change } \\
\text { (low sensitivity, } \\
\text { varies with reagents) }\end{array}$ & $\begin{array}{l}\uparrow(\text { varies with } \\
\text { reagents })\end{array}$ & $\uparrow$ & $\uparrow$ & $\uparrow$ & $\uparrow$ & ND \\
\hline Rivaroxaban & $\begin{array}{c}\uparrow \text { or no change } \\
\text { (not sensitive at low } \\
\text { concentrations, } \\
\text { varies with } \\
\text { reagents }\end{array}$ & $\begin{array}{c}\uparrow \text { or no change (less } \\
\text { sensitive than PT) }\end{array}$ & - & - & - & $\uparrow$ & $\begin{array}{c}\uparrow(\text { sensitive } \\
\text { and specific } \\
\text { when } \\
\text { calibration } \\
\text { curve used) }\end{array}$ \\
\hline Apixaban & $\begin{array}{c}\text { } \text { or no change } \\
\text { (other tests more } \\
\text { sensitive, may vary } \\
\text { with reagents) }\end{array}$ & $\begin{array}{c}\text { } \text { or no change } \\
\text { (other tests more } \\
\text { sensitive, may vary } \\
\text { with reagents) }\end{array}$ & & & & & \\
\hline
\end{tabular}

ND, not detected. Adapted from Siegal and Crowther, $2013 .{ }^{9}$

half-life of 8-11 h. Peak plasma concentrations are similar for both and are reached in approximately 2-4 h. Rivaroxaban and apixaban are mainly metabolized by the liver, in particular by the CYP3A4 system. Studies have reported the efficacy and safety of rivaroxaban for treatment of venous thromboembolism (the EINSTEIN DVT, EINSTEIN PE and EINSTEIN EXTENSION studies $^{10-12}$ ) and several doses were used at start of treatment and in long-term treatment of VTE (15 mg twice daily for 3 weeks followed by $20 \mathrm{mg}$ once daily ).

Double blind randomized trials for treatment of VTE are ongoing also for apixaban (AMPLIFY) and edoxaban (HOKUSAI) but results are not yet available.

\section{Clotting tests to monitor Xa inhibitors}

Although in daily clinical management, and based on their pharmacokinetic and pharmacodynamic profiles, specific or periodic monitoring of anticoagulant activity of new oral anticoagulants is not required, we reported a series of conditions in which anticoagulant activity of new oral anticoagulants is needed and may be helpful in clinical decision making. Several tests are available for rigorous monitoring of intensity of anticoagulant activity of new oral anticoagulants with direct action toward Factor Xa. However, coagulation tests may often have a different sensitivity depending on the blood concentration of Xa inhibitors.

A PT with a plain thromboplastin can be used to determine the relative intensity of anticoagulation due to rivaroxaban (e.g. in an emergency or in an urgent clinical scenario as reported above). On the other hand, each laboratory should be aware of the sensitivity of their PT assay to new oral anticoagulants that are direct inhibitors of Factor $\mathrm{Xa}$, and this can be achieved using commercially available direct Xa-inhibitors plasma calibrants (e.g. rivaroxaban). ${ }^{13,14}$

Several global conventional clotting tests, as well as clotting or chromogenic assays to measure anti-Factor Xa activity, have been studied. Anti-Factor Xa assays (without exogenous antithrombin) and specific PT assays can be used with rivaroxaban plasma calibrants to determine the drug level. The chromogenic tests found a dose-dependent relationship between anti-Factor Xa activity and rivaroxaban concentration. Modified specific Factor Xa chromogenic assays should be investigated in more depth.

A dependent prolongation of PT, dilute PT, and aPTT was observed with rivaroxaban. Results may vary depending on the reagents used and this variation cannot be standardized with the INR system commonly used for vitamin K antagonists. So, results of the PT-test can be expressed in plasma concentrations of rivaroxaban rather than PT seconds or ratio, after which a standard calibration curve can be made. PT was assessed by mixing $50 \mu \mathrm{L}$ of plasma poor platelets at $37^{\circ} \mathrm{C}$ with $100 \mu \mathrm{L}$ of calcium thromboplastin. Several different thromboplastins were tested and reagents from brain rabbit (such as Neoplastin and Neoplastin plus) seem to have a better impact than recombinant human thromboplastin or thromboplastin from placenta. These tests often required special conditions, such as dilute prothrombin time, because in normal PT for common assays and measurements a high concentration of thromboplastin is used (i.e. more thromboplastin is used in routine PT tests than in physiological conditions).

Also rivaroxaban was seen to influence aPTT but the effect was weak. This was to be expected since there is a trend for direct FXa inhibitors to have a 
greater effect on PT than on aPTT. Yet a prolongation of aPTT may be found in particular when the test is performed with kaolin.

\section{IIa inhibitors}

Dabigatran, the main IIa inhibitor, acts by binding to the catalytic site of thrombin. Dabigatran etexilate is its pro-drug and this is available for therapeutic and commercial use. It requires ester cleavage after absorption to be transformed into active form. Its halflife is nearly $12-17 \mathrm{~h}$ after administration of several doses. Dabigatran is then mainly eliminated by kidney $(80 \%)$ and the remaining fractions by liver. The RECOVER, RE-COVER II, RE-MEDY, RE-SONATE ${ }^{15-}$ ${ }^{18}$ studies reported the efficacy and safety of dabigatran for treatment of venous thromboembolism; doses used were $150 \mathrm{mg}$ twice daily.

\section{Clotting tests to monitor IIa inhibitors}

Periodic monitoring of the anticoagulant activity of new oral anticoagulants is also not required for dabigatran. However, when clinical conditions are critical and coagulation tests are required, there are several tests that evaluate the effect of dabigatran. Coagulation tests behave differently with increasing concentration of dabigatran. Of course, the maximum effect of dabigatran on clotting parameters occurs at the same time as maximal plasma concentrations, indicating that thrombin inhibition by dabigatran is a direct effect linked to the central plasma compartment. ${ }^{19}$

Various coagulation assay tests behave differently with increasing concentrations of dabigatran, such as the time curves for activated partial thromboplastin time (aPTT), prothrombin time (PT, expressed as international normalized ratio, INR), thrombin clotting time (TT), ecarin clotting time (ECT). Thrombin inhibitor assay (HEMOCLOT) values parallel the plasma concentration-time curve of dabigatran. ${ }^{20} \mathrm{Of}$ course, when we interpret a coagulation assay, it is essential to know when dabigatran etexilate was administered relative to the time of blood sampling.

The aPTT assay targets the intrinsic pathway of the coagulation cascade. Prolongation of the aPTT occurs with increasing dabigatran plasma concentration although the aPTT concentration-response curve is curvilinear and flattens at higher concentrations ( $\geq 200$ $\mathrm{ng} / \mathrm{mL}$ ). Furthermore, when given to healthy volunteers in supra-therapeutic doses $(400 \mathrm{mg}$ three times daily), aPTT ratios were mostly in the range of 2-3 at trough and peak dabigatran plasma concentrations (>400-500 ng/mL).

The PT assay represents the clotting time in the extrinsic coagulation pathway. Dabigatran has little effect on the PT (INR) at clinically relevant plasma concentrations. Therapeutic concentrations of dabigatran usually result in only modest elevations of INR, while INR rises by 2.0 at supra-therapeutic concentrations of dabigatran.

The TT assay directly assesses the activity of thrombin in a plasma sample and, therefore, directly measures dabigatran activity. The TT is particularly sensitive to the effects of dabigatran and displays a linear dose-response ratio over different therapeutic concentrations. The TT test is readily available in most hospitals.

The ECT assay is a specific assay for thrombin generation. The activator of the assay, ecarin, is a snake venom that specifically activates prothrombin resulting in the generation of meizothrombin, an unstable precursor of thrombin. As DTIs are able to inhibit the thrombin-like activity of meizothrombin, the ECT test provides a direct measure of dabigatran activity.

The Hemoclot ${ }^{\circledR}$ Thrombin Inhibitor assay (Hyphen BioMed, Neuville-sur-Oise, France) is a sensitive diluted TT assay which allows quantitative measurement of DTI activity in plasma, based on inhibition of a constant and defined concentration of thrombin. Mixing normal pooled human plasma and diluted test plasma (clotting is initiated by adding a constant amount of highly purified human alpha-thrombin), we obtain a linear relationship between dabigatran concentration and clotting time (from approx. $30-75 \mathrm{~s}$ ). ${ }^{21}$

\section{Conclusions}

The new oral anticoagulants have shown considerable promise in large-scale, randomized clinical studies for the management of thromboembolic disorders, and have been approved for clinical use in specific indications. These agents offer advantages over VKAs, including rapid onset, shorter half-lives, fewer drug interactions, and lack of need for routine monitoring. However, it is very important to remember that the use of anticoagulant drugs should be carefully monitored clinically, especially in populations at particular risk.

\section{References}

1. Moyer TP, O'Kane DJ, Baudhuin LM, et al. Warfarin sensitivity genotyping: a review of the literature and summary of patient experience. Mayo Clin Proc 2009; 84:1079-94.

2. Hammerstingl C. Monitoring therapeutic anticoagulation with low molecular weight heparins: is it useful or misleading? Cardiovasc Hematol Agents Med Chem 2008;6:282-6.

3. Kearon C, Akl EA, Comerota AJ, et al. American College of Chest Physicians. Antithrombotic therapy for VTE disease: Antithrombotic Therapy and Prevention of Thrombosis, 9th ed: American College of Chest 
Physicians Evidence-Based Clinical Practice Guidelines. Chest 2012;141:419S-94S.

4. Walenga JM, Adiguzel C. Drug and dietary interactions of the new and emerging oral anticoagulants. Int J Clin Pract 2010;64:956-67.

5. Turpie GGA, Kreutz R, Llau J, et al. Management consensus guidance for the use of rivaroxaban - an oral, direct factor Xa inhibitor. Thromb Haemost 2012;108: 876-86.

6. Heidbuchel H, Verhamme P, Alings M, et al. European Heart Rhythm Association Practical Guide on the use of new oral anticoagulants in patients with non-valvular atrial fibrillation. Europace 2013;15:625-51.

7. Dahl OE, Kurth AA, Rosencher N, et al. Thromboprophylaxis with dabigatran etexilate in patients over seventy-five years of age with moderate renal impairment undergoing or knee replacement. Int Orthop 2012; 36:741-8.

8. Huber K, Connolly SJ, Kher A, et al. Practical use of dabigatran etexilate for stroke prevention in atrial fibrillation. Int J Clin Pract 2013;67:516-26.

9. Siegal DM, Crowther MA. Acute management of bleeding in patients on novel oral anticoagulants. Eur Heart J 2013;34:489-98b.

10. Bauersachs R, Berkowitz SD, Brenner B, et al. Oral rivaroxaban for symptomatic venous thromboembolism. N Engl J Med 2010;363:2499-510.

11. Büller HR, Prins MH, Lensin AW, et al. Oral rivaroxaban for the treatment of symptomatic pulmonary embolism. N Engl J Med 2012;366:1287-97.

12. Romualdi E, Donadini MP, Ageno W. Oral rivaroxaban after symptomatic venous thromboembolism: the continued treatment study (EINSTEIN-extension study). Expert Rev Cardiovasc Ther 2011;9:841-4.

13. Samama MM, Contant G, Spiro TE, et al. For the ri- varoxaban anti-factor Xa Chromogenic assay field trial laboratories. Thromb Haemost 2012;107:379-87.

14. Samama MM, Martinoli JL, Le Flem L, et al. Assessment of a laboratory assays to measure rivaroxaban - an oral Xa inhibitor. Thromb Haemost 2010;103:815-25.

15. Schulman S, Kearon C, Kakkar AK, et al. for the RECOVER Study Group Dabigatran versus Warfarin in the Treatment of Acute Venous Thromboembolism. N Engl J Med 2009;361:2342-52.

16. Schulman S, Kakkar AK, Schellong SM, et al. A randomized trial of dabigatran versus warfarin in the treatment of acute venous thromboembolism (RE-COVER II). American Society of Hematology Annual Meeting, San Diego, USA. 2011. Abstract 205

17. Schulman S, Eriksson H, Goldhaber S, et al. Dabigatran or warfarin for extended maintenance therapy of venous thromboembolism RE-MEDY. J Thromb Haemost 2011;9:731-2.

18. Schulman S, Baanstra D, Eriksson H, et al. Dabigatran vs. placebo for extended maintenance therapy of venous thromboembolism RE-SONATE. J Thromb Haemost 2011;9:22.

19. Baetz BE, Spinler SA. Dabigatran etexilate: an oral direct thrombin inhibitor for prophylaxis and treatment of thromboembolic diseases. Pharmacotherapy 2008;28: 1354-73.

20. van Ryn J, Stangier J, Haerrter S, et al. A. Dabigatran etexilate - a novel, reversible, oral direct thrombin inhibitor: Interpretration of coaulation assays and reversal of anticoagulant activity. Thromb Haemost 2010;103: 1116-27.

21. Stangier J, Feuring M. Using the HEMOCLOT direct thrombin inhibitor assay to determine plasma concentrations of dabigatran. Blood Coagul Fibrinolysis 2012; 23:138-43. 